\title{
Relationship between Organized Physical Activity and Motor Competence in Teenagers
}

\author{
Carolina Maria Coelho Campos ${ }^{1,3, *}$, Daniel da Rocha Queiroz ${ }^{2,3}$, Jeymison Tadeu Nunes Da Silva ${ }^{4}$, Anderson \\ Henry P. Feitoza ${ }^{1,3}$, Maria Teresa Cattuzzo ${ }^{1,3,4}$ \\ ${ }^{1}$ Higher School of Physical Education, University of Pernambuco, Recife, Brazil \\ ${ }^{2}$ Department of Physical Education and Sports Science, Federal University of Pernambuco, Vitória de Santo Antão, Brazil \\ ${ }^{3}$ Associate Program of Post-graduation in Physical Education, UPE / UFPB, Recife, Brazil \\ ${ }^{4}$ Post-graduation Program in Hebiatrics, UPE, Recife, Brazil \\ *Corresponding author: carolccampos@hotmail.com
}

\begin{abstract}
It is not clear if the association between motor competence (MC) and organized physical activity (OPA) in teenagers and if it depends on the gender. The purpose of this study was to investigate relationship between MC and OPA by gender. Participants, 29 male (19.3 \pm 1.3 years) and 38 female (19.0 01.2 years) were assessed by the BOT-2 brief form and by a retrospective questionnaire of the OPA. Males had superior performance in body coordination, strength, agility, and the general motor competence. There were no significant differences between genders in the time spent in OPA; there was a positive and low correlation between MC and OPA, but the correlation showed stronger values only for females. OPA seems to be an enabling environment for the acquisition and development of motor skills that provide the foundation for engagement and motor competence, but gender is an interaction variable.
\end{abstract}

Keywords: psychomotor performance, motor activity, adolescent

Cite This Article: Campos, C.M.C; Queiroz, D.R.; Silva, J.T.; Feitoza, A.H.P.; Cattuzzo, M.T. Relationship between Organized Physical Activity and Motor Competence in Teenagers. American Journal of Sports Science and Medicine, vol. 5, no. 4 (2017): 82-85. doi: 10.12691/ajssm-5-4-4.

\section{Introduction}

Organized physical activity (OPA) such a participation in sports, dance and martial arts, is considered one of the most important environment for youth to improve levels of physical activity [1]. OPA involves regular classes or training, are structured under a adult supervision (i.e. coach, instructor, or teacher) [2] and have been related with raising the time spent in moderate to vigorous physical activity and decreasing the youth's health risk [1,3]. Previous studies show that individuals engaged in OPA are more likely to achieve the physical activity guidelines [4], have greater levels of PA [3] and spend more time in moderate, vigorous [4], when compared to non-participants. Thus, understand the variables related with OPA represent a promising strategy for enhancing youth's health.

The literature has showed a positive relationship between motor competence (MC) and OPA $[5,6,7,8]$ during the life span. Studies like Queiroz et al. [8], in early childhood, and Vandorpe et al. [6] and D'Hondt et al. [7], in middle childhood, found that individuals with higher levels of $\mathrm{MC}$ where more engaged on OPA, when compared with those with lower levels of MC. However, few studies have reported the relationship between OPA and MC in older ages $[9,10]$ including gender analyses.

The literature indicates that a large part of the adolescents has a low level of positive behaviors for an improvement of their health [11] and that the relationship between MC and OPA can be different between boys and girls [12]. Results from Okely et al. [12] showed that the relationship between $\mathrm{MC}$ and time spent in OPA was stronger in girls when compared with boys. However, few studies have analyzed a relationship between MC and OPA and its own moderating effect between the genders.

The potential difference between gender could give insight into different cultural practices (such as encouragement in some types of sport regarding gender e.g., soccer for boys) that could help or hinder development in certain types of skills or make one gender spend more time in OPA when compared with the other [13]. Clearly, gender studies can provide valuable understanding into how different motor skills developed in different gender and how it related with OPA [13]. Although cross-sectional studies do not imply causation, correlational researches allow determining if there is a relationship between the variables, adding to gaps in the literature. Thus, the aim of this study was to analyze the relationship between $\mathrm{MC}$ and $\mathrm{OPA}$ in adolescents, specifically to analyze gender differences between MC and OPA of the adolescents.

\section{Methods}

\subsection{Study Design and Participants}

The present cross sectional study assessed a convenient sample of 67 healthy Brazilians adolescents (boys $=29$ ), from 17 to 21 years old, recruited from an urban university in 
northwest of Brazil with similar social demographic status. Individuals with any physical disability or health condition that prevented completion of the testing were not included in the sample.

\subsection{Procedures}

Prior to the study, written consent from parents was obtained for all participants and the study was approved by the local committee. All participants had initially their anthropometrics variables assessed. Body mass was measured by portable scale (Glicomed - nearest $0.1 \mathrm{~kg}$ ) and height was measured by the portable stadiometer scale (GIMI - nearest $0.1 \mathrm{~cm}$ ).

\subsubsection{Organized Physical Activity}

Information about the OPA was obtained through a self-reported questionnaire by Campos et al. [14]. Individuals were asked to reported the type of the OPA (e.g. swimming, soccer, ballet), frequency of participation (i.e., times attended per week) and the duration (i.e., hours per practice) of the activities in which they were engaged. All questionnaires were completed at the facility at the university under a trained research assistant supervision. OPA defined as those activities that involved regular classes, training, or competition; were formally structured and had a coach, instructor, or teacher [14]. Examples include attending dance or martial arts; swimming or athletics clubs; playing soccer or basketball or any other teams sport. A self-reported measurement, is often the only feasible methodology that can be utilized to address the research questions of this particular interest, it is cost and time efficient, easy to administer, and has been used in many studies investigating participation in organized sport participation $[5,12,14]$, even for a recall of past experiences [14]. Additionally, the questionnaire was tested with a sample of non-participating students of the same ages and from the same school, with no issues of clarity reported, and presenting excellent values of validity (content validity index (CVI), based on experts' ratings of item relevance, clarity and pertinence. $\mathrm{CVI}=85 \%$ ) and excellent reliability (Cohen's kappa coefficient $\geq .75$ ). In order to know the adolescents global experience in OPA, in this study used the total time spent in OPA in minutes.

\subsubsection{Motor Competence}

Motor competence was assessed by Bruininks-Oseretsky Test of Motor Proficiency - Second Edition, Brief Form [16]. This is an individually administered test that uses engaging, goal-directed activities to measure a wide array of motor skills in individuals aged 4 through 21 . The
BOT-2 brief form contains 12 items in 4 domains: 1) fine manual control; 2) manual coordination; 3) body coordination and 4) strength and agility. The completion time was approximately of 15 minutes, realized in free disturbance, and closed environment according with the protocol test. Test was administered by well trained examiners and intra and inter-rater, the percent agreement was above $85 \%$. This study used the raw scores of each domain and the total motor composite (sum of all domains).

\subsection{Data Analyses}

Normal distribution of variables was tested by Kolmogorov-Smirnov test. Mann-Whitney $U$ test was used to analyze the difference between means, Cohen's effect size was carried out by Thalheimer and Cook [17] protocol; Spearman correlation was calculated to examine associations between MC and OPA and was also carried out by gender. The strength of associations was defined as: $0.10-0.25=$ low; $0.26-0.49=$ moderate and $0.50-1.0=$ strong [18]. An alpha $<.05$ was implemented to determine statistics significances. SPSS (version 10.0) was used for all data analyses.

\section{Results}

Initial analysis to identify the inter-rater reliability on the motor competence assessment showed high levels of reliability among researchers (intra $=92 \%$; inter-rater $=90 \%$ ). Table 1 shows the anthropometric characteristics of the participants by gender. Results showed that there were no significant different between teenagers by age, height and weight.

The motor performance is categorized by gender and summarized in Table 2. Mann-Whitney U Test confirmed there was a significant difference between teenagers with boys presenting a superior performance in two different motor skill's domains (body coordination and strength and agility) and also in the total motor composite score, when compared with girls. There was no significant difference at the time spent in OPA among the teenagers (Table 2).

Table 1. Mean (M) and standard deviation (SD) of age (years), high (cm) and weight $(\mathrm{kg})$ of the participants by gender.

\begin{tabular}{lccc}
\hline & Boys (n=29) & Girls (n=38) & \\
\cline { 2 - 4 } & M (SD) & M (SD) & $p$ \\
\hline Age (years) & $19.3(1.3)$ & $19.0(1.2)$ & 0.13 \\
Height $(\mathrm{cm})$ & $173.9(6.1)$ & $161.9(5.7)$ & 0.09 \\
Weight $(\mathrm{kg})$ & $74.5(11.2)$ & $53.3(8.7)$ & 0.41 \\
\hline
\end{tabular}

Table 2. Mean (M) and standard deviation (SD) of motor proficiency and the time spent in organized physical activity by gender.

\begin{tabular}{lccccc}
\hline & \multicolumn{2}{c}{ Boys (n=29) } & \multicolumn{3}{c}{ Girls (n=38) } \\
\hline & M & SD & M & SD & $p$ \\
Organized physical activity (min/week) & 82944.8 & 81779.2 & 75343.8 & 56222.7 & .167 \\
Fine Manual Control (pts) & 16.1 & 1.9 & 16.6 & 1.6 & .365 \\
Manual Coordination (pts) & 12.5 & 1.0 & 12.6 & 0.9 & .715 \\
Body Coordination (pts) & 21.5 & 2.7 & 19.6 & 3.6 & .035 \\
Strength and Agility (pts) & 5.8 & 1.9 & 3.5 & 1.3 & $<.001$ \\
General Motor Skill (pts) & 56.1 & 4.1 & 52.5 & 4.7 & .006 \\
\hline
\end{tabular}


Spearman results showed in general there was a positive and low correlation between time spent in OPA and $\mathrm{MC}(r h o=0,252, p=0,039)$. When stratified by gender the results described an interaction effect in the correlation between time spent in OPA and MC, showing a positive and moderate correlation only for girls (rho $=0,323, p=0,048)$.

\section{Discussion}

This study aimed was to analyze the relationship between $\mathrm{MC}$ and OPA in adolescents. I was verified the differences among boys and girls $\mathrm{MC}$ and the relationship between $\mathrm{MC}$ and OPA of the adolescents, considering the gender. Our study found there was a significant difference between the $\mathrm{MC}$ of the teenagers, and this was already expect, once the literature reports evidences with similar results $[19,20]$, although there is no consensus about which gender presents the better MC. In our results, boys had a superior performance when compare with girls, and this is similar with found by some studies [20,21] but the opposite found by Gidley Larson et al., when analyzing children [19].

According to Ré [22] there is no difference between boys and girls until adolescence. After that, there will be a morphological and functional difference, extremely related with the hormonal changes that could affect the motor performance. However, the same author also highlighted that, if there was an adequate involvement in sports and PA the motor performance of the adolescent, especially girls, could not be declined. The ambiguous results reported by the literature would be likely related with the difference of opportunities to practice, causing a lack of motor learning and $\mathrm{MC}$ in boys and girls [8]. Thus, even our results reporting a better performance of boys, is plausible that it could be reversed if girls had the opportunity to practice the motor skill leading their improvement of performances. The correlations results also support this point, since it was found that MC score was positive and significantly related with time spent in OPA, although the value of the correlation was low. Okely et al. [12] corroborated by Fransen et al. [5] also reported that those adolescents who had more time spent in OPA also had better MC when compared with those with less time. Likewise, in their study with students in the 7 grade, Jaakkola et al. [23] founded the organized physical activity in sport clubs was more strongly associated with MC than was not-organized physical activity during leisure.

The explanation is that OPA is a kind of physical activity which provides an important environment for motor learning, once it promotes not only the physical effort but also the cognitive effort to attend the instruction, feedback and other practical arrangements ideal for the motor learning and, consequently, improve MC [24,25]. Also, demands in OPA lies on the development of the percepto motor/neuromuscular system, i.e., the ability to recruit motor units, to increase motor unit firing rates, to have an optimal co-activation of agonist/antagonist muscles, and synergistic muscle contractions, which is critically important to the development of MC [26]. So, it is possible to suggest the environment which involves OPA take on both informational and energetic challenges required to improve the $\mathrm{MC}$. Also, the relationship between $\mathrm{MC}$ and musculoskeletal and cardiorespiratory fitness becomes more reciprocal in nature during late childhood and adolescence [27].

It was identified an interaction effect in the correlation between MC and OPA with girls presenting the stronger value than boys. This result is similar with found by Okely et al. [12] who also reported a stronger correlation between MC and OPA for girls when compared with the boys, which was explained by the authors with the fact that boys have also more social acceptance in OPA practice when compared with girls. This means that regardless their MC boys would be more engaged in PA and OPA because they have the social approval, but the girl's involvement in the OPA might be considered more socially unacceptable and this could address for an engagement in OPA only for those who achieved skilled movements [12].

Our results also reported there was no difference in the time spent in OPA of the boys and girls. This could indicate initially a contradiction in this discussion, once it was assumed that same opportunity to practice motor skill would lead to similar results of MC. However, two different aspects should be considered about the relationship between $\mathrm{MC}$ and practice. The first one is that, the present study assessed only the amount of time spent in OPA. According to Holfelder \& Scott [28], boys can clearly spend more time in non-organized PA than girls, which also improves the levels of $\mathrm{MC}$, once the development of skill is also promoted by frequent activation of neural pathways [29]. Thus, even the amount of time spent in OPA was similar between the teenagers in this study; it was possible that the amount of time spent in non organized PA could be responsible for the boys' superior levels of MC.

The second aspect that should consider is that the same amount of time for practice does not necessary means same quality of practice. Not only the amount of minutes spent, but also the amount of time spent with appropriate training and supervision (which include instruction and feedback) is considered the key for the motor development [30]. Lemos, Avigo and Barela [31] reported that subject who had physical education class supervised by a physical education teacher had superior MC when compared with those without a trained teacher, which support the importance of the quality spent in practice that aim improvement in $\mathrm{MC}$ rather just the amount spent.

Some limitations should be considered when interpreting the findings of this study. The sample not randomly selected and included only one high school limiting generalizability. Further, the cross-sectional nature of the data limits the ability determine a causal relationship.

\section{Conclusions}

Although a correlational study cannot prove that changes to one variable lead to changes to another variable, evidence from correlation studies can lead to testing that evidence under causal conditions since correlational study are the stepping-stone to the more powerful method. Thus, the results of this study can suggest that individuals who were engaged in OPA had an adequate scenario for develop their $\mathrm{MC}$ and that gender was considered an interaction variable in this relationship. 
Our results support the importance of practice of motor skill in a trained and supervised environment and also that girls might need a different encouragement for their initiation and maintenance in OPA. Promoting and sustaining positive OPA trajectories in youth by specifically targeting the development of $\mathrm{MC}$ is a unique approach and has the potential to significantly impact activity levels across lifespan.

\section{Acknowledgements}

This work was supported by the state Funding Agency FACEPE under grant IBPG-0721-4.09/12 and Coordenação de Aperfeiçoamento de Pessoal de Nível Superior (CAPES) under Grant 6392-15-6.

\section{References}

[1] Malina RM. Children and adolescents in the sport culture: the overwhelming majority to the select few. J Exerc Sci Fit 2009; 7(2): S1-S10.

[2] Fraser-Thomas J, Côté J. Youth Sports: Implementing Findings and Moving Forward with Research. J Sport Psychology 2006; 8(3): 12-27.

[3] Wickel EE, Eisenmann JC. Contribution of youth sport to total daily physical activity among 6-to 12-yr-old boys. Med Sci Sport Exer 2007; 39(9): 1493-1500.

[4] Marques A, Ekelund U, Sardinha LB. Associations between organized sports participation and objectively measured physical activity, sedentary time and weight status in youth. J Sci Med Sport 2016; 19(2): 154-157.

[5] Fransen J, Pion J, Vandendriessche J, Vandorpe B, Vaeyens R, Lenoir $\mathrm{M}$, et al. Differences in physical fitness and gross moto coordination in boys aged 6-12 years specializing in one versus sampling more than one sport. J Sport Sci 2012; 30(4): 379-386.

[6] Vandorpe B, Vandendriessche J, Vaeyens R, Pion J, Matthys S, Lefevre J, et al. Relationship between sports participation and the level of motor coordination in childhood: A longitudinal approach. J Sci Med Sport 2012; 15(3): 220-225.

[7] D'Hondt E, Deforche B, Gentier I, De Bourdeaudhuij I, Vayens R, Philippaerts R, et al. A longitudinal analysis of gross motor coordination in overweight and obese children versus normalweight peers. Int J Obesity 2013;37(1): 61-67.

[8] Queiroz DR, Ré AHN, Henrique RS, Moura MS, Cattuzzo MT. Participation in sports practice and motor competence in preschoolers. Motriz 2014; 20(1): 26-32.

[9] O’Neill JR, Williams HG, Pfeiffer KA, Dowda M, McIver KL, Brown WH, et al. Young children's motor skill performance: Relationships with activity types and parent perception of athletic competence. J Sci Med Sport 2014; 17(6): 607-610.

[10] Ridgway CL, Ong KK, Tammelin TH, Sharp S, Ekelund U, Jarvelin MR. Infant motor development predicts sports participation at age 14 years: northern Finland birth cohort of 1966. Plos One 2009; 4(8): e6837.

[11] Barufaldi LA, Abreu GDA, Coutinho ESF, Bloch KV. Metaanalysis of the prevalence of physical inactivity among Brazilian adolescents. Cad Saude Publica 2012; 28(6): 1019-1032.
[12] Okely AD, Booth ML, Patterson JW. Relationship of physical activity to fundamental movement skills among adolescents. Med Sci Sport Exer 2001; 33(11): 1899-1904.

[13] Bardid F, Rudd JR, Lenoir M, Polman R, Barnett LM. (2015) Cross-cultural comparison of motor competence in children from Australia and Belgium. Front Psychol 2015; 6: 964.

[14] Campos, CMC, Oliveira, DS, Feitoza, AHP, Cattuzzo, MT. (2017). Reliability and content validity of the organized physical activity questionnaire for adolescents. Educational Research, 8(2), 021026.

[15] Forsman H, Grasten A, Blomqvist M, Davids K, Liukkonen J, Konttinen N. Development and validation of the perceived gamespecific soccer competence scale. J Sport Sci 2016; 34(14): 1-9.

[16] Bruininks RH, Bruininks BD. BOT2: Bruininks-Oseretsky Test of Motor Proficiency: Administration Easel. Pearson Assessments; 2005.

[17] Thalheimer W, Cook S. How to calculate effect sizes from published research: A simplified methodology. Work-Learning Research 2002: 1-9.

[18] Cohen J. A power primer. Psychol Bull 1992; 112: 155-159.

[19] Gidley Larson JC, Mostofsky SH, Goldberg MC, Cutting LE, Denckla MB, Mahone EM. Effects of gender and age on motor exam in typically developing children. Dev Neuropsychol 2007; 32(1): 543-562.

[20] Chung LMY, Chow LPY, Chung JWY. Normative reference of standing long jump indicates gender difference in lower muscular strength of pubertal growth. Health 2013; 5(6): 6-11.

[21] Moreno-Briseño P, Díaz R, Campos-Romo A, Fernandez-Ruiz J. Sex-related differences in motor learning and performance. Behav Brain Funct 2010; 6(1): 74.

[22] Ré AHN. Crescimento, maturação e desenvolvimento na infância e adolescência: Implicações para o esporte. Motricidade 2011; 7(3): 55-67

[23] Jaakkola T, Kalaja S, Liukkonen J, Jutila A, Virtanen P, Watt A. Relations among physical activity patterns, lifestyle activities, and fundamental movement skills for Finnish students in grade 7. Percept Motor Skill 2009; 108(1): 97-111.

[24] Lee TD, Swinnen SP, Serrien DJ. Cognitive effort and motor learning. Quest 1994; 46(3): 328-344.

[25] Guadagnoli MA, Lee TD. Challenge point: a framework for conceptualizing the effects of various practice conditions in motor learning. J Motor Behav 2004; 36(2): 212-224.

[26] Stodden DF, Goodway JD, Landgendorfer SJ, Roberton MA, Rudisill ME, Garcia C, et al. A Developmental Perspective on the Role of Motor Skill Competence in Physical Activity: An Emergent Relationship. Quest 2008; 60(2): 290-306.

[27] Cattuzzo MT, Henrique RS, Ré AHN, Oliveira IS, Melo BM, Moura MS, et al. Motor competence and health related physical fitness in youth: a systematic review. J Sci Med Sport 2016; 19(2): 123-129.

[28] Holfelder B, Schott N. Relationship of fundamental movement skills and physical activity in children and adolescents: A systematic review. Psychol Sport Exerc 2014; 15(4): 382-391.

[29] Hamstra-Wright KL, Swanik CB, Sitler MR, Swanik KA, Ferber $\mathrm{R}$, Ridenour $\mathrm{M}$, et al. Gender comparisons of dynamic restraint and motor skill in children. Clin J Sport Med 2006; 16(1): 56-62.

[30] Cook HD, Kohl, HW. Educating the Student Body: Taking Physical Activity and Physical Education to School. National Academies Press; 2013.

[31] Lemos AG, Avigo EL, Barela JA. Physical education in kindergarten promotes fundamental motor skill development. Advances in Physical Education 2012; 2(01): 17-2. 